\title{
Are clusters of mental retardation correlated with clusters of developmental delay?
}

\author{
Huiling Zhen ${ }^{1}$, Suzanne McDermott ${ }^{2}$, Andrew B. Lawson ${ }^{3}$, C. Marjorie Aelion ${ }^{4}$ \\ ${ }^{1}$ Department of Epidemiology and Biostatistics, University of South Carolina, South Carolina, Columbia, USA; \\ ${ }^{2}$ Department of Family and Preventive Medicine, University of South Carolina, South Carolina, Columbia, \\ USA; ${ }^{3}$ Division of Biostatistics and Epidemiology, College of Medicine, Medical University of South Carolina, \\ South Carolina, Columbia, USA; ${ }^{4}$ School of Public Health, University of Massachusetts, Amberst, USA
}

\begin{abstract}
Mental retardation (MR) is a subset of developmental delay (DD), a broader classification of childhood disability. The purpose of this study was to determine if clusters of these two conditions were statistically significantly correlated. The residential addresses of 81,935 Medicaid insured pregnant women during each month of pregnancy were used to identify clusters of MR and DD in their children. Correlations between MR and DD were computed based on the sets of P-value surface from selected centroid points, where the P-value for cumulative relative risk of MR and DD was known. The correlations are quite small for all the 10 gestational months for which maternal addresses were available, but they are all statistically significant. This indicates MR and DD are correlated, but they are not linear. When MR was used as the centroid point to identify a cluster the only correlations that were statistically significant were for gestational month 5 and 6 with correlation $0.14(\mathrm{P}=0.007)$ for both months. When the centroid points were selected based on the significance of risk of DD, the correlations between MR and DD are not statistically significant for any month. Correlation between MR and DD based on the sets of P-value surfaces from $4 \mathrm{MR}$ clusters are significant in gestational month 5, 6 and 7 with correlation $0.17(\mathrm{P}=0.047), 0.16(\mathrm{P}=0.060)$ and $0.17(\mathrm{P}=0.044)$, respectively. Our finding suggests that locations of high risk for the more severe condition, MR, also identify a spatial area where less severe cases of DD might be present, however the reverse is not the case.
\end{abstract}

Keywords: mental retardation, developmental delay, spatial clusters, South Carolina.

\section{Introduction}

Childhood developmental disabilities are an epidemiological challenge because their risk factors are only partially understood and there are limited tools to study associations. One area of inquiry that has not been extensively investigated is the association of maternal exposures to environmental toxins with the child outcome using spatial statistics. In order to study spatial associations between maternal expo-

\footnotetext{
Corresponding author:

Suzanne McDermott

Department of Family and Preventive Medicine

University of South Carolina

South Carolina, Columbia, USA

Tel. +1 803434 2445; Fax +1 8034348374

E-mail: Suzanne.mcdermott@uscmed.sc.edu
}

sures during pregnancy and child outcomes we need information about maternal residence during pregnancy. Clusters of the child outcome can be identified using residential addresses of pregnant women which can be linked to child outcomes.

One of the first steps in investigating clusters is the development of a case definition of the outcome. The labeling of childhood disabilities in the USA is directed by the national education legislation, Individuals with Disabilities Education Act (IDEA), which defines "developmental delays (DD), as measured by appropriate diagnostic instruments and procedures, in one or more of the following areas: physical development, including vision and hearing; communication development; social or emotional development; adaptive development; or a diagnosed physical or mental condition that has a 
high probability of resulting in developmental delay" (IDEA, 2004). Mental retardation (MR) is the largest of the 13 disability categories (autism, deaf-blindness, emotional disturbance, hearing impairment, mental retardation, multiple disabilities, orthopedic impairment, other health impairment, specific learning disability, speech and language impairment, traumatic brain injury, visual impairment, deafness) included under DD (National Dissemination Center for Children with Disabilities, 2007). In this study DD will exclude MR so the two categories are mutually exclusive. MR and DD usually have prenatal origins even though the effects are not identified until infancy or childhood. A substantial literature has focused on the prevalence of these conditions, since prenatal incidence can rarely be detected (McDermott et al., 2007). Although there is an assumption of a normal distribution of intelligence in the population, there is evidence of variation in rates of MR across spatial domains as documented in national registries and small area analyses (Westerinen et al., 2007). The explanation for spatial variation has focused on economic and demographic characteristics of the women and children in these localities.

Almost all diagnosed conditions for which there are addresses of cases and controls can be analyzed to determine if there are clusters. MR and DD are no exceptions. Clusters are defined as spatial areas with "unusually increased disease incidence rates". The term "hot spot" has been used to define a cluster if an "unusual" high rate is found (Lawson, 2006a). Cluster detection methods used in spatial epidemiology have used both regional count data as well as case-control data, with controls selected from at-risk populations in order to estimate the relative risk or local rates (Kulldorff, 1997).

MR and DD are the most prevalent childhood conditions, however, there are no previous analyses of spatial or spatio-temporal clustering of these conditions using individual case data. For some time environmental exposures have been suspected as a possible risk factor for unknown etiology cases of MR and DD, but the methods to detect an associa- tion have been limited to conventional epidemiological approaches.

Identification of MR and DD clusters based on maternal residence during pregnancy can provide an important tool to investigate risk exposures since environmental exposures to chemicals during pregnancy have been suspects for damaging brain development in utero (Zhen et al., 2008). It is possible that maternal exposure to contaminated soil, water or air could be associated with neurodevelopmental processes in the development of a fetus. For this reason we designed a study to explore the monthly residential data to see if residence of mothers during a specific month of pregnancy was associated with excess risk for the diagnosis of MR or DD. It is well established that poverty is associated with risk for both MR and DD and that people live in neighbourhoods based on their income. Thus there have been problems inherent with studying the geographic clustering of MR and DD.

This study was designed to reduce the bias of income clustering of families by using data from only low income mothers and children. More specifically, this study was designed to determine if clusters of the MR or DD outcomes varied by gestational month of maternal residence, for women and children insured by Medicaid in a state with coverage included pregnant women up to $185 \%$ of federal poverty levels and children up to $100 \%$ of poverty. In this paper we examine the correlation between clusters of MR and DD which are identified by using a Bayesian hierarchical modeling technique.

\section{Materials and methods}

The present study is a retrospective cohort study which includes 81,935 maternal child pairs to identify clusters of MR and DD cases in a region of South Carolina (SC), USA. The study is designed to identify associations between chemicals in the soil and risk for MR and DD. We received Medicaid approval to conduct the study and University of South Carolina institutional review board (IRB) 
exempt approval. The Medicaid data use agreement required masking of the actual location of cases and controls so address coded data were offset before the research team received the codes. Because of this masking of location we cannot identify actual cluster areas but we could pool the data from risk areas to identify suspect chemicals. The focus of this report is on the first step in the process, when we identified clusters.

Maternal and child Medicaid data from inpatient and outpatient medical reimbursement records which include diagnostic codes and birth certificates records were linked for the women who were pregnant during the period January 1, 1996 through December 31, 2001. Addresses were obtained for each month of pregnancy and geocoded to determine the exact monthly location of the mothers who were pregnant during the study period. After geocoding the locations were offset by the Medicaid contractor who supplied the data for the research project. Ten gestational months were used instead of nine since pregnancy can extend beyond 40 weeks of gestation and although the first and tenth month had a smaller proportion of mothers, we included all the Medicaid pregnancies during the period for a region of the state.

Mothers entered the dataset throughout the months of pregnancy and other mothers dropped out. Entry was based on obtaining Medicaid eligibility and drop outs resulted from moving away, pregnancy loss, and change in eligibility status. Thus, during each month of pregnancy we have a different denominator. Most of the pregnant women entered the Medicaid programme in months 2-4. There was movement of mothers in our study cohort. In fact $22 \%$ moved once, $2 \%$ moved twice and $0.1 \%$ moved more than twice during pregnancy. In addition there is movement out of Medicaid throughout childhood as the income eligibility changes for families. During pregnancy Medicaid is the most inclusive with families eligible up to 1.85 times higher than the federal poverty level. Since children with a disability qualify for Medicaid we expect there will be higher retention of children with
MR compared to children without a disability.

The Medicaid reimbursement files for the pregnant mothers, birth certificate data, hospital and outpatient care for both the mother, and the child health records through 2007 were merged. As a result we had maternal prenatal files and child records for 6-10 years following birth to identify codes for MR and DD in the Medicaid record.

\section{Case definition}

The identification of cases of MR and DD in this analysis included two important steps. The strategy was designed to identify all cases of MR and DD, and then exclude those that had a known genetic, infectious, injury or alcohol related cause. We wanted to focus on the cases with unknown cause since this is the group for whom an environmental exposure could be a risk factor. First we identified a list of known causes of MR and DD, and their ICD9 codes using multiple sources (WHO, 2002). We excluded 2754 babies with a known cause of MR/DD. The excluded infants had one of the following known causes of MR/DD: Trisomy 13, 1618, other chromosomal aberrations, Prader-Willi Syndrome, Rett's Syndrome, phenylketonuria, Fragile X Syndrome, postnatal injury, prenatal rubella, meningitis, encephalitis and Fetal Alcohol Syndrome.

We then defined two mutually exclusive groups. Children who had an ICD9 code for MR were classified as having MR, even if they also had a DD code in some part of their record. Children with DD never had a MR code so the two groups were mutually exclusive. The infants and children with MR were identified by the use of ICD9 code 317 (mild MR), 318 (moderate and severe MR), or 319 (unknown severity MR) in the Medicaid inpatient or outpatient/private practice record. Through this process we identified 3003 cases of MR (3.9\%). Identification of DD was based on use of the ICD9 code of 315 . Through this process we identified 11584 cases of DD (14.9\%).

The denominator for the identification of clusters 
was all children born during the study period who were insured by Medicaid.

\section{Cluster detection}

Recently a novel approach to the detection of spatial clusters was proposed for both count data in small areas and also case event data at residential addresses (Hossain and Lawson, 2005; Lawson, 2006b). This method is based on Bayesian models for the cluster form. They rely on the definition of a likelihood which is a function of a "lasso" parameter. This parameter defines the size of an area within which cases are accumulated. Both cases of disease and control cases can be accumulated in the lasso. In the situation where binomial data are found (such as where we have children with and without MR), we simply count the number of MR cases within the lasso and also the number of children without MR (as control cases). The likelihood used is a local likelihood based on the lasso found for each site. The lassos can and do overlap. These local likelihood methods are designed to provide estimates of areas of excess risk across the continuous disease risk surface of the map. When the algorithm is implemented the posterior distribution of lassos is sampled over a large number of iterations and the posterior average lasso for each area is obtained. As a function of this lasso the relative risk is also obtained. The local relative risk is important in cluster detection as it is this risk that can show where excesses of the case disease are found. We assume that our clusters are essentially "hot spots" in any area of the map which shows unusually high risk. The posterior average relative risk is computed and also the "significance" of the local risk elevation is assessed by the estimation of an exceedence probability. This probability is defined as $p_{i}=P r$ $\left(R R_{i}>1\right)$ where $R R_{i}$ is the local relative risk at the $i$-th site. This is computed from the posterior sample of relative risks found for each site. To match up with the usual criteria for significance tests, we examine $q_{i}=1-p_{i}$ for local areas with $q_{i}<0.05$ or $<0.01$. These areas can be considered as unusual or significantly elevated. Note that our model provides a description of the spatial variation of risk on the map over all areas and also we do not need to adjust for multiple testing, unlike other methods such as SaTScan.

The advantages of this method is that: (i) it produces a continuous risk map where gradients of risk are apparent as well as clusters; (ii) it does not limit the clusters to circular shapes; and (iii) it automatically allows the incorporation of covariates within a full likelihood formulation. The final output from the local likelihood sampler is in the form of a P-value surface. This surface can then be contoured or displayed as a heat image or perspective plot and any (possibly irregular) areas of highly excessive risk will be represented by areas below the 0.05 or 0.01 contour levels.

\section{Statistical analyses}

The characteristics of the Medicaid study population in the clusters with MR and DD were analyzed using chi-square tests for categorical variables and $t$ test for continuous variables to determine if there were statistically significant differences in the proportions within each of the categories or if there were differences in the means (Table 1).

The spatial clusters were identified by using Bayesian local likelihood model based on count data in small areas instead of case event data at residential address (Devine et al., 1996; Hossain and Lawson, 2005; Lawson, 2006b). Data conversion from individual observation at residential address to count data in small areas was performed using the ArcGIS 9.3 software. The first step identified appropriate geographic coordinate system/units for the data, and the boundary file points formed a converted polygon for the area. Equal size grid meshes were created as fine as possible within the boundary, and ended up with a total of 5088 grid cells with each grid cell size $3500 \times 3500 \mathrm{~m}$. A centroid location of each grid cell was extracted, and numbers of MR cases, DD cases and controls in each grid cell were counted. For the centroid location of each grid cell, the local relative 
risk of MR with corresponding P-value, and the local relative risk of $\mathrm{DD}$ with corresponding $\mathrm{p}$-value were calculated, respectively, based on Markov chain Monte Carlo (MCMC) local likelihood sampling techniques, which was performed by FORTRAN programme. Then pixellated heat image maps with colors corresponding to the P-values were created for $\mathrm{MR}$ and DD separately using the R programme, and MR clusters and DD clusters were identified based on contour graphs in 10 gestational months.

Five Pearson's correlations between MR and DD were computed based on the P-value surface from different sets of centroid locations:

(i) P-value surface from all the 5088 centroid locations;

(ii) P-value surface from the centroid locations where the local relative risk of MR has statistically significant P-value $(<0.05)$, while the local relative risk of DD may or may not have statistically significant P-value;

(iii) P-value surface from the centroid locations where the local relative risk of DD has statistically significant P-value, while the local relative risk of MR may or may not have statistically significant P-value;

(iv) P-value surface from the centroid locations of four MR cluster areas; and

(v) P-value surface from the centroid locations of four DD cluster areas.

The normality assumption of Pearson correlation was examined by histograms, and scatter plots of Pvalue of excess risk of MR versus P-value of excess risk of DD were created when the Pearson correlations were statistically significant. The Pearson correlations, normality assumption test, and scatter plots were performed using SAS version 9.1 statistical software (Cary, NC, USA).

\section{Results}

The mean or proportion of some of the known characteristics associated with MR and DD for the study population in the MR cluster areas is shown in Table 1. In MR cluster areas, children with MR are more likely to be preterm ( $<28$ weeks gestation), have no sibling, have lower birth weight, and have mothers who are older at the time of birth compared to the children with DD; and there is no evi-

Table 1. Mean or proportion of the characteristics for the study population.

\begin{tabular}{|c|c|c|c|c|c|}
\hline \multirow[b]{2}{*}{ Variable } & & \multicolumn{2}{|c|}{ Mean/N (proportion) } & \multirow[b]{2}{*}{ Test statistics } & \multirow[b]{2}{*}{ P-value } \\
\hline & & MR & DD & & \\
\hline $\begin{array}{l}\text { Birth weight } \\
\text { Mean grams at birth }\end{array}$ & & 3027 & 3198 & -4.94 & $<0.001$ \\
\hline $\begin{array}{l}\text { Mother's age } \\
\text { Mean years }\end{array}$ & & 24.03 & 22.75 & 3.11 & 0.002 \\
\hline $\begin{array}{l}\text { Baby sex } \\
\text { Number (\%) }\end{array}$ & $\begin{array}{l}\text { boy } \\
\text { girl }\end{array}$ & $\begin{array}{l}266(63.5) \\
153(36.5)\end{array}$ & $\begin{array}{l}875(60.6) \\
569(39.4)\end{array}$ & 1.14 & 0.285 \\
\hline $\begin{array}{l}\text { Alcohol } \\
\text { Number (\%) }\end{array}$ & $\begin{array}{l}\text { yes } \\
\text { no }\end{array}$ & $\begin{array}{r}4(1.0) \\
413(99.0)\end{array}$ & $\begin{array}{r}9(0.6) \\
1431(99.4)\end{array}$ & 0.52 & 0.471 \\
\hline $\begin{array}{l}\text { Weeks gestation } \\
\text { Number }(\%)\end{array}$ & $\begin{array}{r}>36 \text { week } \\
28-36 \text { weeks } \\
<28 \text { weeks }\end{array}$ & $\begin{array}{r}292(76.8) \\
75(19.7) \\
13(3.4)\end{array}$ & $\begin{array}{r}1099(84.2) \\
198(15.2) \\
9(0.7)\end{array}$ & 22.55 & $<0.001$ \\
\hline $\begin{array}{l}\text { Mother's race } \\
\text { Number }(\%)\end{array}$ & $\begin{array}{r}\text { White African } \\
\text { American } \\
\text { Other }\end{array}$ & $\begin{array}{r}176(42.0) \\
237(56.6) \\
6(1.4)\end{array}$ & $\begin{array}{r}685(47.4) \\
746(51.7) \\
13(0.9)\end{array}$ & 4.45 & 0.108 \\
\hline $\begin{array}{l}\text { Parity } \\
\text { Number (\%) }\end{array}$ & $\begin{array}{r}0 \\
1 \\
2 \\
\geq 3 \\
\end{array}$ & $\begin{array}{r}172(41.1) \\
129(30.8) \\
55(13.1) \\
63(15.0) \\
\end{array}$ & $\begin{array}{r}559(38.7) \\
497(34.4) \\
249(17.2) \\
139(9.6) \\
\end{array}$ & 13.87 & 0.003 \\
\hline
\end{tabular}


dence to show that there are any differences between children with MR and children with DD in terms of gender, mother's race, and mother's report of alcohol use during pregnancy.

The contour maps of MR and DD are displayed side by side in Figures 1 to 6 , which are plotted at 0.05 and 0.1 of P-value of excess risk of MR and $\mathrm{DD}$, and are based on gestational month $5-$ month
7 data. The clusters are defined as "hot spots" in any area of the map which shows significant high risk. The MR clusters are identified from Figure 1, 3 and 5, and the DD clusters are identified from Figures 2, 4 and 6. The number of MR clusters identified is much less than that for DD, which may be due to the reason that case event of MR is much less than DD in our study area. Furthermore, the MR
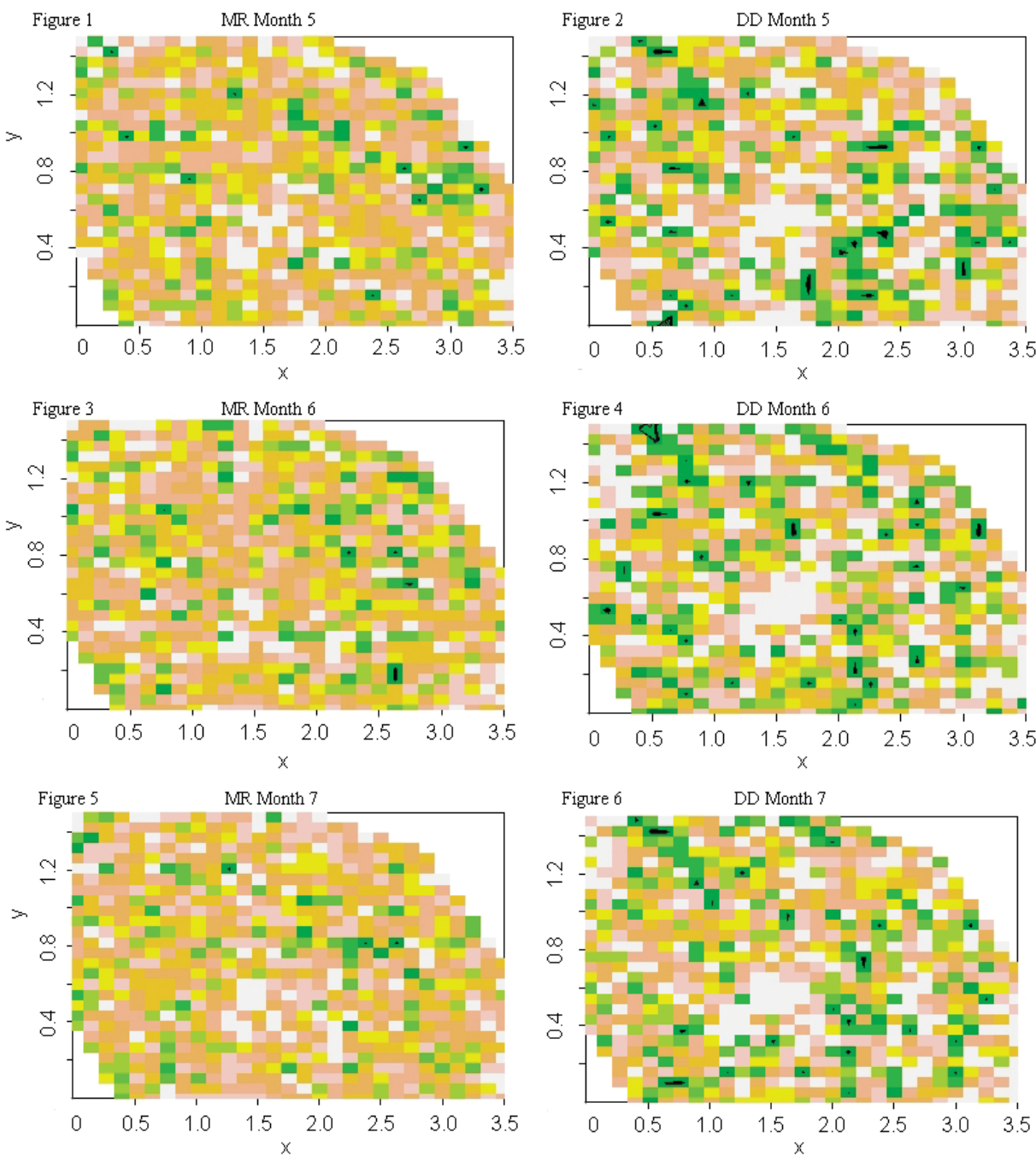

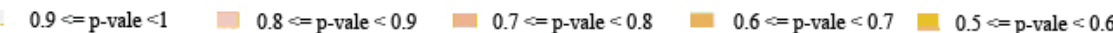

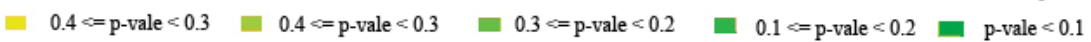

Figs. 1-6. Contour maps of MR and DD, side by side, plotted at $\mathrm{P}=0.05$ and $\mathrm{P}=0.01$ excess risk for MR and DD, gestational month 5 - month 7 (0.5 units in xaxis indicate 28.6 miles, 0.4 units in y-axis indicate 28 miles). 
clusters identified are very close to or in the same areas as some of the DD clusters. The 0.5 units on the $\mathrm{x}$-axis are 28.6 miles and the units on the $\mathrm{y}$-axis are 28 miles.
Five different Pearson's correlations between MR and DD were computed based on the P-value surface from different sets of centroid locations to show if there are same hot spots for both MR and

Table 2. Correlations between MR and DD.

\begin{tabular}{|c|c|c|c|c|c|c|c|c|c|c|}
\hline $\begin{array}{l}\text { Gestation } \\
\text { month }\end{array}$ & 1 & 2 & 3 & 4 & 5 & 6 & 7 & 8 & 9 & 10 \\
\hline \multicolumn{11}{|c|}{ Correlation $1 *$} \\
\hline $\mathrm{N}$ & 5088 & 5088 & 5088 & 5088 & 5088 & 5088 & 5088 & 5088 & 5088 & 5088 \\
\hline Correlation & 0.0385 & 0.0386 & 0.0388 & 0.0327 & 0.0242 & 0.0406 & 0.0918 & 0.0483 & 0.0290 & 0.0500 \\
\hline $\begin{array}{l}\text { P-value of } \\
\text { correlation }\end{array}$ & 0.006 & 0.006 & 0.006 & 0.020 & 0.085 & 0.004 & $<0.001$ & $<0.001$ & 0.039 & $<0.001$ \\
\hline \multicolumn{11}{|c|}{ Correlation $2 *$} \\
\hline $\mathrm{N}$ & 279 & 347 & 360 & 349 & 357 & 369 & 357 & 345 & 358 & 184 \\
\hline Correlation & 0.0880 & 0.0360 & 0.0079 & 0.0916 & 0.1424 & 0.1394 & 0.0750 & 0.0756 & 0.0929 & 0.0924 \\
\hline $\begin{array}{l}\text { P-value of } \\
\text { correlation }\end{array}$ & 0.143 & 0.504 & 0.882 & 0.088 & 0.007 & 0.007 & 0.157 & 0.161 & 0.079 & 0.213 \\
\hline \multicolumn{11}{|c|}{ Correlation $3^{*}$} \\
\hline $\mathrm{N}$ & 756 & 868 & 998 & 1012 & 1090 & 1109 & 1048 & 1052 & 965 & 565 \\
\hline Correlation & 0.0374 & 0.0152 & 0.0112 & 0.0463 & 0.0349 & 0.0007 & 0.0348 & 0.0593 & 0.0329 & 0.0419 \\
\hline $\begin{array}{l}\text { P-value of } \\
\text { correlation }\end{array}$ & 0.304 & 0.655 & 0.725 & 0.141 & 0.249 & 0.982 & 0.261 & 0.055 & 0.307 & 0.320 \\
\hline \multicolumn{11}{|c|}{ Correlation $4 *$} \\
\hline$\overline{\mathrm{N}}$ & 141 & 141 & 141 & 141 & 141 & 141 & 141 & 141 & 141 & 141 \\
\hline Correlation & 0.0110 & 0.1012 & 0.0005 & 0.1001 & 0.1677 & 0.1587 & 0.1703 & 0.1040 & 0.0755 & 0.0154 \\
\hline $\begin{array}{l}\text { P-value of } \\
\text { correlation }\end{array}$ & 0.897 & 0.233 & 0.996 & 0.238 & 0.047 & 0.060 & 0.044 & 0.220 & 0.374 & 0.856 \\
\hline \multicolumn{11}{|c|}{ Correlation $5^{*}$} \\
\hline $\mathrm{N}$ & 238 & 238 & 238 & 238 & 238 & 238 & 238 & 238 & 238 & 238 \\
\hline Correlation & 0.0631 & 0.0683 & 0.1713 & 0.0123 & 0.0244 & 0.0055 & 0.0160 & 0.0344 & 0.0479 & 0.0245 \\
\hline $\begin{array}{l}\text { P-value of } \\
\text { correlation }\end{array}$ & 0.333 & 0.294 & 0.008 & 0.850 & 0.708 & 0.933 & 0.807 & 0.598 & 0.462 & 0.707 \\
\hline
\end{tabular}

$\mathrm{N}=$ number of observations used in the correlation calculation.

Correlation $1^{*}=$ correlations are based on the P-values of all 5088 centroid points in Northern SC.

Correlation $2 *$ correlations are based on the P-values of selected centroid points, where Medicaid MR has P-values less than 0.05 and Medicaid DD may or may not have P-value less than 0.05 .

Correlation $3 *$ = correlations are based on the P-values of selected centroid points, where Medicaid DD has P-values less than 0.05 and Medicaid MR may or may not have P-value less than 0.05.

Correlation $4 *$ = correlations are based on the P-values of selected centroid points, from four Medicaid MR clusters.

Correlation $5^{*}=$ correlations are based on the P-values of selected centroid points, from four Medicaid DD clusters. 
DD. Table 2 shows the correlations for each of 10 gestational months. Correlation 1 was computed based on the sets of P-value surface from all the 5088 centroid points. The correlations are quite small for all the 10 gestational months, but they are all statistically significant. This indicates there is a correlation between MR and DD, but they are not linear. Correlation 2 between MR and DD was computed based on the sets of P-value surface from the selected centroid points, where the P-value for cumulative relative risk of $\mathrm{MR}$ is significant $(\mathrm{P}<0.05)$ while the $\mathrm{P}$-value for the cumulative relative risk of DD may or may not be significant. The correlations are statistically significant for gestational month 5 and 6 with correlation $0.142(\mathrm{P}=$ $0.007)$, and 0.139 ( $\mathrm{P}=0.007)$, respectively. The correlations for all other gestational months are not statistically significant. Correlation 3 between MR and DD was computed in a reverse way as it did in correlation 2, that is the centroid points were selected based on the significance of P-value of cumulative relative risk of DD, not MR. The correlations between MR and DD calculated in this way are not statistically significant for all the 10 gestational months. Correlation 4 between MR and DD was computed based on the sets of P-value surface from the selected centroid points which are from four MR clusters. The correlations calculated in this way are significant in gestational month 5, 6 and 7 with correlation $0.168(\mathrm{P}=0.047), 0.159$ $(\mathrm{P}=0.060)$ and $0.170(\mathrm{P}=0.044)$, respectively. The correlations for other gestational months are not statistically significant. Correlation 5 between MR and DD was computed in a reverse way as it did in correlation 4, that is the centroid points were selected from the 4 clusters of DD, not MR. The correlations between MR and DD calculated in this way are not statistically significant for all the 10 gestational months.

\section{Discussion}

This study was possible because we had data for each month of maternal residence for pregnant women insured by the Medicaid programme and the medical data for their children. Half of all births in SC are insured by Medicaid and these children represent the highest risk group for MR and DD due to factors associated with poverty.

We found the correlation between risk gradients in Medicaid identified MR and Medicaid identified DD were statistically significant for gestational months 5, 6 and 7 when the correlations were based on centroid points within four identified MR clusters (correlation 4 in Table 2). The only other condition in which correlations were statistically significant was when the correlations were based on the P-values of selected centroid points where Medicaid MR had a P-value less than 0.05 (correlation 2 in Table 2).

One of the statistical strengths of this investigation is the large sample size. Our original data are case event data of residential addresses with large sample sizes, which require huge computation time and cause the identified clusters to be unstable. Therefore, without loss of the details, we used count data in the cluster analysis process instead of case event data of residential addresses. Significance of Pearson correlation can be influenced by the normality assumption and outlier. The normality assumptions were tested by comparing histograms of P-values of excess risk of MR and DD with the normal distributions, and no substantial differences were found. Furthermore, it is well accepted that normality assumption will not be a major concern if the sample size is large than 100, while sample size for each of gestational month in our study is much larger than 100 . Therefore, violation of normality assumption for correlation is not an issue in our study.

Another statistical feature of this investigation is the spatial clusters were identified by using a Bayesian local likelihood model. A large number of cluster detection approaches have been proposed and applied in many fields, such as Tango's maximized excess events test (MEET), spatial scan statistic and Bayesian local likelihood model. Tango's MEET statistic is evaluated using Monte Carlo hypothesis testing and has good power in detecting global clustering but it does not work very well for 
rural clusters (Song and Kulldorff, 2003). The spatial scan statistic imposes a circular window on the map and lets the circle centroid move across the study region. The spatial scan statistic has good power in detecting hot spot clusters but it restricts the identified cluster shape to a circle (Song and Kulldorff, 2003). Bayesian local likelihood modeling used in this investigation is a very useful tool to identify MR and DD clusters. It allows flexible cluster forms and the incorporation of covariates. It provides a description of the spatial variation of risk on the map over all areas, and we do not need to adjust for multiple testing, unlike other methods such as SaTScan.

The methodological strength of this investigation includes the ability to only use mother-child pairs who were insured by Medicaid so the bias of poverty is addressed. In addition, some of the issues related to the validity of MR and DD diagnosis that plague other studies are minimized in this investigation. The use of address codes to identify clusters is not based on residence when the diagnosis of MR was made; instead it is based on maternal residence during pregnancy. Thus the issues of bias of some physician's use of the ICD9 codes for the diagnosis of MR would impact studies that rely on child addresses, but is much less an issue when maternal pregnancy addresses are used.

One of the limitations of the exploratory stage of this study is that, as yet, we have not examined the association of clusters in MR outcome and associated explanatory variables. Thus the identified relationship between geography of maternal residence is operating through one of these risk factors. Our future work may extend to the joint analysis of MR and DD and explore the shared and divergent trends (Dabney and Wakefield, 2005) in risk between these two disease.

In conclusion, this investigation allowed us to compare the risk level for MR and DD at specific maternal residential locations, during each month of gestation. This was done in cluster areas where the observed MR rate is $10 \%$ higher the rate for the larger region. We found that when Medicaid MR is used to define the locations of significant risk then Medicaid DD risk levels had a correlation of $17 \%$ for gestational months $5(\mathrm{P}=0.047)$, a correlation of $16 \%$ for month 6 (marginally significant $\mathrm{P}=0.060)$ and a correlation of $17 \%$ for month 7 $(\mathrm{P}=0.044)$ (Table 2). When Medicaid DD is used to define the locations of significant risk then gestational month 8 was the only statistically significant month $(\mathrm{P}=0.055)$. This finding suggests that locations of high risk for the more severe and lifelong condition, MR, also identifies a spatial area where less severe cases of DD might be present, however the reverse is not the case. The identification of irregular shaped clusters of $\mathrm{MR}$ can be used to direct research into potential environmental risks.

\section{Acknowledgments}

Funding for this research was provided by the National Institutes of Health, National Institute of Environmental Health Sciences, RO1 Grant No. ES012895-01A1.

\section{References}

Devine OJ, Louis TA, Halloran ME, 1996. Identifying areas with elevated disease incidence rates using empirical Bayes estimators. Geogr Anal 28, 187-199.

Dabney AR, Wakefield JC, 2005. Issues in the mapping of two diseases. Stat Met Med Res 14, 83-112.

Hossain M, Lawson AB, 2005. Local likelihood disease clustering: development and evaluation. Environ Ecol Stat 12, 259-273.

Individuals with Disabilities Education Improvement Act (IDEA), 2004. http://thomas.loc.gov/cgi-bin (accessed on 16 June 2008).

Kulldorff M, 1997. A spatial scan statistic. Comm Stat 26, 1481-1496.

Lawson AB, 2006a. Statistical Methods in Spatial Epidemiology. Wiley, New York, USA.

Lawson AB, 2006b. Disease cluster detection: a critique and a Bayesian proposal. Stat Med 25, 897-916.

McDermott S, Durkin MS, Schupf N, Stein Z, 2007. Epidemiology and etiology of mental retardation. In: Jacobson JW, Mulick, JA, Rojahn J (Eds). Handbook of 
Intellectual and Developmental Disabilities. Springer Press, New York, USA.

National Dissemination Center for Children with Disabilities, 2007. www.nichcy.org (accessed on 28 June 2008).

Song C, Kulldorff M, 2003. Power evaluation of disease clustering tests. Int J Health Geogr 2, 9.

Westerinen H, Kaski M, Virta L, Almqvist F, Iivanainen M,
2007. Prevalence of intellectual disability: a comprehensive study based on national registers. J Intell Dis Res 51, 715-725. WHO, 2002. International statistical classification of diseases, 2002, 9th ed. Geneva, Switzerland.

Zhen H, Lawson AB, McDermott S, Aelion CM, 2008. Spatial analysis of mental retardation and residence during pregnancy. Geospat Health 2, 173-182. 